\title{
Electrochemical Impedance Spectroscopic Analysis of ZnS Nanorod Fabricated Using Butterfly Wings as Biotemplate
}

\author{
K. Jeyasubramanian $\cdot$ M. Nisanthi $\cdot$ V. S. Benitha $\cdot$ N. Selvakumar
}

Received: 23 July 2013/Revised: 19 April 2014/Published online: 18 November 2014

(C) The Chinese Society for Metals and Springer-Verlag Berlin Heidelberg 2014

\begin{abstract}
This article describes the growth of zinc sulfide ( $\mathrm{ZnS}$ ) nanorod on glass/aluminum foil by employing butterfly wings as biotemplate. Upon calcinating (at $400{ }^{\circ} \mathrm{C}$ ), the butterfly wings soaked in $\mathrm{ZnS}$ nanoparticle suspension, with uniform cage-like nanostructures in nanodimensions, were found on glass/aluminum surface. The transverse and longitudinal dimensions of the nanorods were evaluated from scanning electron microscopy micrographs as 132 and $159 \mathrm{~nm}$, respectively. Purity of the $\mathrm{ZnS}$ nanorod found on the specimen was checked by recording XRD $\left(28.877^{\circ}, 48.038^{\circ}\right.$, and $\left.57.174^{\circ}\right)$ and Fourier transform infrared spectrometer spectra $\left(663.7\right.$ and $551.68 \mathrm{~cm}^{-1}$ ). Luminescence natures of the nanorods were examined using photoluminescence spectral studies. The characteristic emission peak is shown in the visible region with strong intensity, while the excitation peak is shown at $267 \mathrm{~nm}$. Electrochemical impedance spectroscopic analysis of $\mathrm{ZnS}$ nanorod exhibits double-layer capacitance value $\left(C_{\mathrm{dl}}=6.7 \mathrm{nF}\right)$, and the Bode plot explains the stability of $\mathrm{ZnS}$ nanorod under the influence of electrical field.
\end{abstract}

KEY WORDS: Semiconducting material; Biotemplate; Photo luminescence (PL); Nanorod; Bode plot; Capacitance

\section{Introduction}

Nanostructured materials are a new class of materials, having dimensions in nanoranges, which possess improved performance with extended capabilities of products in a wide range of applications [1]. Nanostructures with mono disperse size and shape have unique optical and physical properties compared with bulk materials. Based on the nanostructure shapes, they can be classified into three types, namely, zerodimensional, one-dimensional (1D), and two-dimensional (2D) materials. 1D nanostructures represent the smallest

Available online at http://link.springer.com/journal/40195

K. Jeyasubramanian $(\bowtie) \cdot$ M. Nisanthi ·

V. S. Benitha · N. Selvakumar

Centre for Nanoscience and Technology, Department of

Mechanical Engineering, Mepco Schlenk Engineering College,

Sivakasi 626005, India

e-mail: kjeya@mepcoeng.ac.in dimension structure that can efficiently transport electrical carriers. The different types of the nanostructures can be synthesized in the purest form with the different controlled environments, such as chemical composition, temperature, concentration of precursors, etc. The ability to produce 1D nanostructures with controlled dimensions allows making functional nanoscale devices [2].

Among various nanomaterials, semiconductor nanomaterials, especially metal chalcogenide materials, have been studied for the optical and electronic properties since they are associated with quantum confinement effect. As an important wide band gap II-VI group semiconducting material, zinc sulfide $(\mathrm{ZnS})$ is a promising candidate with $3.65 \mathrm{eV}$ [3]. It attracted many researchers, since it is having varieties of applications in photoconductors, flat panel displays, electroluminescent devices, light emitting diodes (LEDs), solar cell, infrared windows, sensors, lasers, biodevices, etc. [4-6]. ZnS nanobelts have been utilized as UV—light sensors, UV—light photo detectors by many 
researchers [7-9]. They are also anticipated to play an important role in the interconnects and functional units in fabricating electronic and optoelectronic devices. Some methods, such as sonochemical method, sol-gel technique, solvothermal method, template method, etc., have been reported recently for the preparation of $\mathrm{ZnS}$ nanocrystallites [10]. Among the different types of fabrication techniques, controlling the size and shape is a critical issue. However, the shape and size of $\mathrm{ZnS}$ nanorods in required dimensions have been achieved by modifying various parameters [11-13]. Biotemplate-assisted technique is a common technique which allows for fabrication of nanorod, nanobelt, hollow spheres, etc.

The wings of the butterflies are composed primarily of cuticle, a multilayered material consisting of chitin microfibers embedded in a protein matrix [14]. The cuticle is arranged in the form of tubular, supporting veins and thin deformable membranes. Veins found on the wings are naturally micro-capillary tubular structures, through which fluid medium containing nanoparticles can enter by capillary movement [15]. This study makes a novel attempt in the loading of $\mathrm{ZnS}$ nanoparticles in the hollow veins present on the wings of the butterflies, to develop the nano$\mathrm{ZnS}$ cages with cross sections in nanometer size range on aluminum foil. The nano-ZnS presenting in the suspension easily penetrated into the hollow veins through capillary action. Precursor-loaded butterfly wings on calcination at moderate temperature yields pure $\mathrm{ZnS}$ nanorods mimicking the cage-like arrangements found in butterfly wings.

The structural properties of the $\mathrm{ZnS}$ nanorods developed over glass and $\mathrm{Al}$ foil were investigated by $\mathrm{X}$-ray diffraction technique, scanning electron microscopy, FTIR analysis, etc. The electrical property of $\mathrm{ZnS}$ is measured by electrochemical impedance spectroscopy (EIS). The capacitance value $\left(C_{\mathrm{dl}}\right)$ and electron life time $\left(\tau_{\mathrm{n}}\right)$ of $\mathrm{ZnS}$ nanostructures were evaluated through EIS.

\section{Experimental}

\subsection{Materials}

Zinc acetate and sodium sulfide as a source material used in this work were purchased from E-Merck Ltd., India, and used without purification.

\subsection{Preparation of $\mathrm{ZnS}$ Nanorod}

$0.1 \mathrm{~mol}$ of zinc acetate and $0.1 \mathrm{~mol}$ of sodium sulfide were dissolved in $50 \mathrm{~mL}$ of distilled water separately and mixed slowly by constant stirring. The $\mathrm{ZnS}$ nanoparticles are formed during the addition of sodium sulfide into the zinc acetate solution as white precipitate. The $\mathrm{ZnS}$ nanoparticles separated were removed by centrifugation, and each particle is repeatedly washed with distilled water for removing the unreacted impurities. Then, the $\mathrm{ZnS}$ in wet condition was poured into $70 \mathrm{~mL}$ of distilled water, sonicated for $30 \mathrm{~min}$ to get a $\mathrm{ZnS}$ dispersed suspension. The cleaned and dried butterfly wing was soaked in the suspension for $30 \mathrm{~min}$, removed, and dried in air. After drying, the wings were placed in between two glass slides. It looks like a sandwich model. The glass slide was kept at $400{ }^{\circ} \mathrm{C}$ for about $1 \mathrm{~h}$ in muffle furnace. Biological materials like chitin, cuticle, etc., upon calcination were burnt out leaving the nanorod pattern of $\mathrm{ZnS}$. Similarly, ZnS nanorods were also grown over aluminum foil by employing the same technique. Before placing the precursor-loaded butterfly wings, the glass slide was covered using a cleaned aluminum foil. Remaining $\mathrm{ZnS}$ nanoparticles found in the aqueous suspension were separated as nanopowder by centrifuging with the supernatant medium. The obtained nanorods made of $\mathrm{ZnS}$ were used for further analysis.

\subsection{Electrochemical Impedance Spectroscopy}

The EIS has been widely used for studying the electrochemical mechanisms occurring at electrode/electrolyte interface, and it establishes an equivalent electrical circuit model for the electrochemical processes [16]. The Nyquist plot gives the information about impedance value and the capacitance values of semiconducting material, whereas from Bode plot, the charge carrier life time was evaluated.

From Nyquist plot, the capacitance $\left(C_{\mathrm{dl}}\right)$ of $\mathrm{ZnS}$ is calculated using the following equation:

$C_{\mathrm{dl}}=\frac{1}{2 \pi f Z^{\prime \prime}}$,

where $f$ is the frequency of the applied electric potential signal; $Z^{\prime \prime}$ is the phase component of the measured impedance.

$Z^{\prime \prime}$ value was calculated from the maximum of real part of impedance multiplied by the angle (between the line and the X-axis) of the impedance graph.

The life time $\left(\tau_{\mathrm{n}}\right)$, known as an average time of excited electron residing on the conduction band before recombining to the hole, was calculated from Bode plot obtained from impedance analysis [17]:

$\tau_{\mathrm{n}}=\frac{1}{2 \pi f_{\max }}$,

The maximum frequency $\left(f_{\max }\right)$ was calculated from the phase plot.

\subsection{Characterization Techniques}

Crystal parameter of the nanoparticle and nanorods were analyzed using an X-ray diffractometer (Philips X'pert pro 
$\mathrm{X}$ ray diffractometer) by $\mathrm{Cu} K_{\alpha 1}(\lambda=1.54056 \AA)$ radiation by measuring the $2 \theta$ values varied from $15^{\circ}$ to $60^{\circ}$ at scanning rate of $0.05 \%$. The confirmation of compounds was studied by Fourier transform infrared spectrometer (FTIR Alpha-Bruker optics, Germany). The morphology of the sample was characterized using a scanning electron microscope (SEM) Model: SU1510, Hitachi, Japan. The surface characteristic was studied by atomic force microscopy (AFM) (Park System South Korea-XE70). The photo luminescence spectra were measured by exciting the specimen with the wavelength of $267 \mathrm{~nm}$ using a $150-\mathrm{W}$ xenon lamp as an excitation source (Shimadzu spectrometer-RF 530 IPC, Japan). The impedance spectroscopy of the samples was recorded using Electrochemical workstation CHI 660C, USA.

\section{Results and Discussion}

\subsection{FTIR Study}

FTIR spectrum of $\mathrm{ZnS}$ powder is presented in Fig. 1. The infrared spectrum is formed due to the absorption of electromagnetic radiation at the frequencies, which correlates to the vibration of chemical bonds that exist within a molecule. The spectrum exhibits characteristic vibrations for $\mathrm{Zn}$ and $\mathrm{S}$ at 663.7 and $552.68 \mathrm{~cm}^{-1}$, respectively [18]. A peak at $1,416.62 \mathrm{~cm}^{-1}$ is attributable to the $\mathrm{C}-\mathrm{H}$ band's presence in the wings of butterfly in trace amounts as organic contents like chitin, cuticle, etc. Another peak appearing at $1,555.67 \mathrm{~cm}^{-1}$ is due to the $\mathrm{C}-\mathrm{O}-\mathrm{H}$ stretching vibrations of organic contents [18].

\subsection{XRD Analysis}

Figure 2a represents the XRD pattern of $\mathrm{ZnS}$ nanopowder synthesized by co-precipitation method. The XRD of ZnS nanorod obtained by biotemplate method is shown in Fig. 2b. Both XRD patterns have three major peaks at $2 \theta$ values of $28.877^{\circ}, 48.038^{\circ}$, and $57.174^{\circ}$ corresponding to a diffraction planes of (101), (111), and (022) planes, respectively (JCPDS no: 892425), which correspond to the

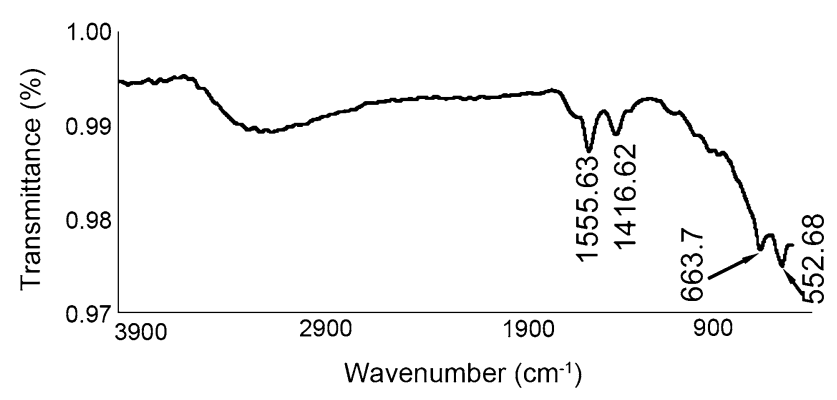

Fig. 1 FTIR spectrum of ZnS nanorod hexagonal structure [19]. The XRD patterns of ZnS nanorod and nanopowder do not show any other peaks, confirming that the samples are free from impurities. The crystallite sizes of the $\mathrm{ZnS}$ nanopowders and nanorods were calculated using Scherer equation [20], and the measured crystallite sizes are 65.93 and $39.948 \mathrm{~nm}$, respectively.

\subsection{SEM Analysis}

Figure 3 shows the typical SEM images of $\mathrm{ZnS}$ nanorods grown on glass slide using biotemplate method. Figure $3 \mathrm{a}$ shows well-organized porous hierarchical architecture of the $\mathrm{ZnS}$ nanorod at low magnification. Maximum length of the nanorod found vertically is about $3 \mu \mathrm{m}$, while that of the horizontal nanorod is more than $10 \mu \mathrm{m}$. Such a longsized nanostructure fabrication is possible only with the help of biotemplate method, and not by other methods [21]. A higher magnification image in Fig. 3b clearly exhibits the parallel ridges that are aligned by stack of the multilayer, and it is possible to determine the dimensions of longitudinal and transverse directions. SEM observation demonstrates that the diameters of the nanorods found in longitudinal and transverse directions are 159 and $132 \mathrm{~nm}$, respectively.

\subsection{AFM Studies}

Atomic Force Microscopy (AFM) image of the $\mathrm{ZnS}$ nanorod is shown in Fig. 4. The nanotextured structure is well explored by this image, and it shows 3D image of $\mathrm{ZnS}$ nanorod [22]. The $\mathrm{ZnS}$ nanorods were observed on the glass substrate, where it is possible to identify the groups of the nanorods with similar dimensions and nearly the same orientation. The structural features of the nanorod have been explored by the XEI-image processing software supplied by PARK system, South Korea. As-grown ZnS nanorod appears as a thick cover over the aluminum foil with a thickness of about $400-500 \mathrm{~nm}$.

\subsection{Photoluminescence (PL) Properties}

Luminescence is a characteristic property of semiconducting materials, the absorption and emission characteristics of which are size dependent [23-25]. The information obtained from the PL spectra is useful to detect and analyze the electronic states of the $\mathrm{ZnS}$ nanopowder and nanorod. The PL spectra of the as-synthesized $\mathrm{ZnS}$ nanopowder and the asgrown $\mathrm{ZnS}$ nanorod are shown in Fig. 5. Strong emission peaks found at about 365 and $476 \mathrm{~nm}$ (For ZnS nanopowder) are revealed on exciting the specimen using 267-nm source radiation. The exciton peak around $365 \mathrm{~nm}$ indicates that, it is a near-band edge emission that originated due to the recombination of excitons of $\mathrm{ZnS}$ nanopowder [26]. The broad band 

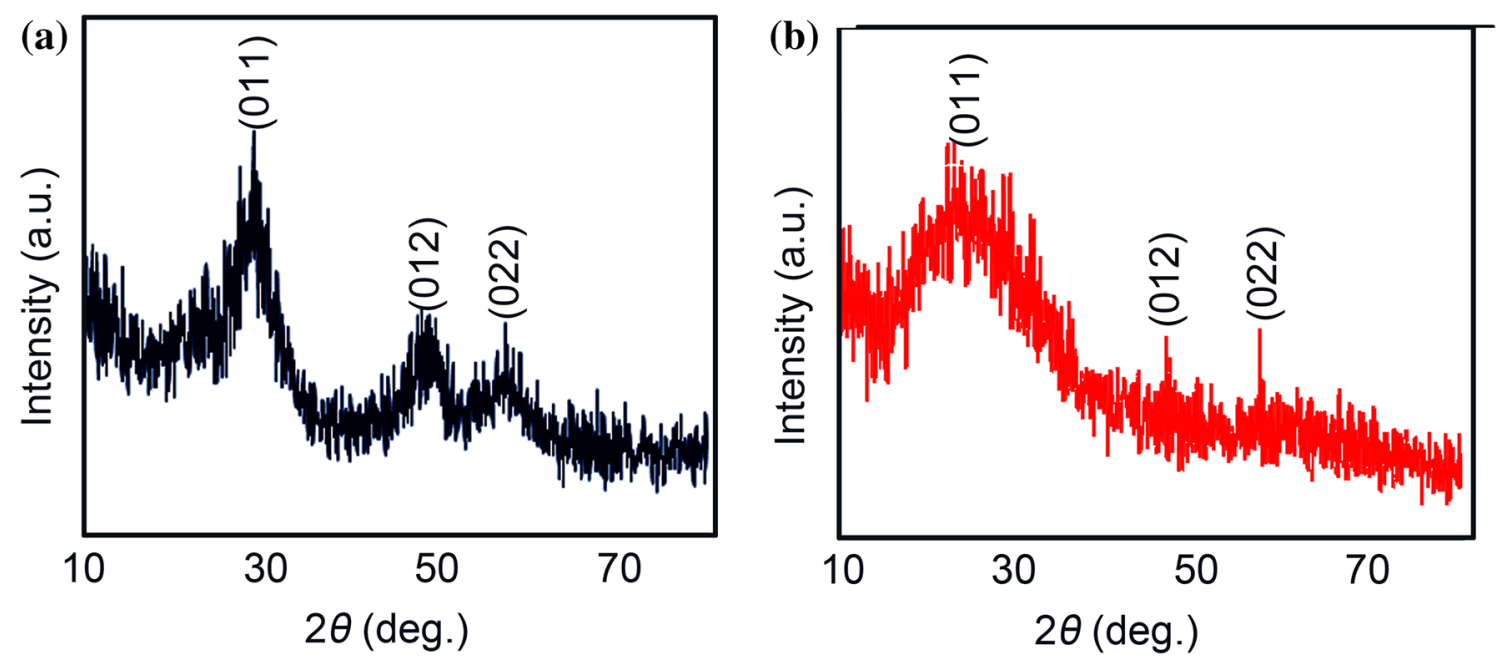

Fig. 2 XRD patterns of $\mathrm{ZnS}$ nanopowder a, $\mathrm{ZnS}$ nanorod $\mathbf{b}$
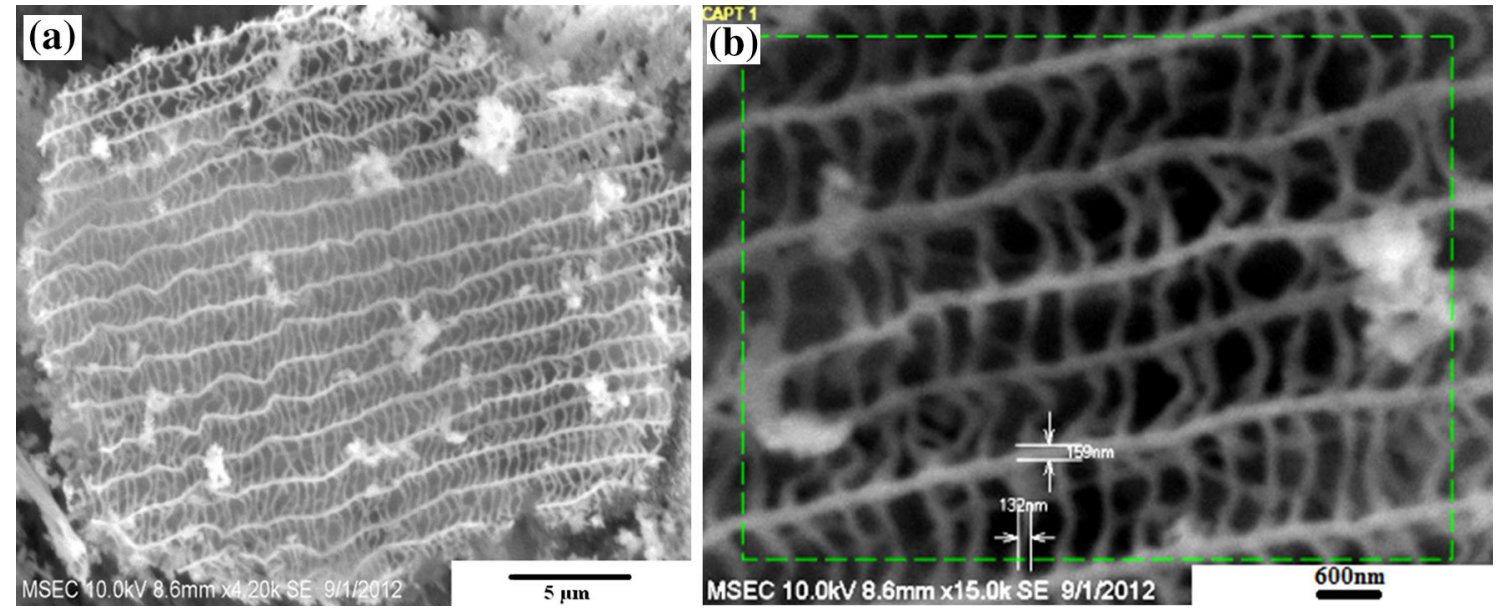

Fig. 3 SEM images of ZnS nanorod with low a, high magnifications $\mathbf{b}$

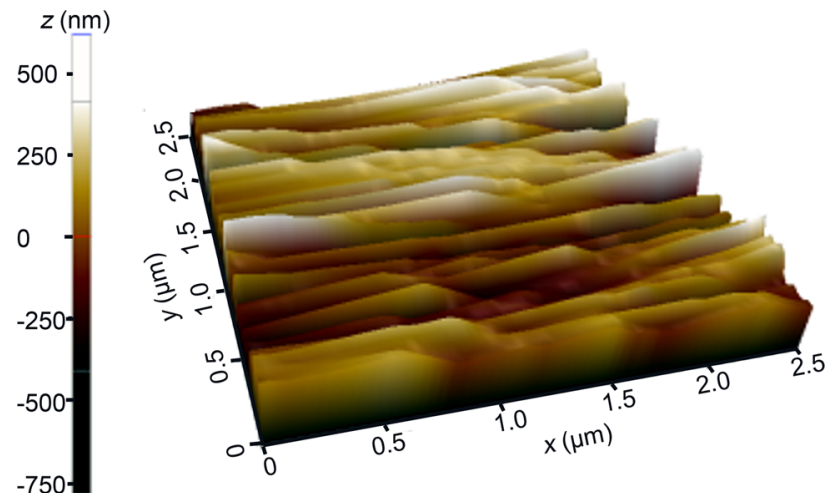

Fig. 4 AFM image of $\mathrm{ZnS}$ nanorod

emission at $476 \mathrm{~nm}$ is attributed to the stoichiometric vacancies or interstitial impurities. Compared to that of $\mathrm{ZnS}$ nanopowder, $\mathrm{ZnS}$ nanorod exhibits the band edge emission at $360 \mathrm{~nm}$ (blue shift). The precise weak peak found at $452 \mathrm{~nm}$ is attributable to interstitial impurities existing in the nanorod [27]. Another broad band originating from the nanorod located at $470 \mathrm{~nm}$ is assigned to stoichiometric vacancy or interstitial impurities. The $\mathrm{ZnS}$ nanorods have shorter emission wavelength than that of the $\mathrm{ZnS}$ nanopowder. The concentration of the defects increases with the increasing degree of crystallinity. The emission intensity characteristic to nanopowder is high since it is associated with higher percentage of stoichiometric vacancy or interstitial impurities. At the same time, $\mathrm{ZnS}$ nanorod displays a low-intense spectrum attributed to the defect-free structures [28].

\subsection{EIS Analysis}

Thin film of semiconducting materials possesses electrical properties such as A.C impedance, charge transport, etc. [29, 30]. All such behaviors have been measured through 


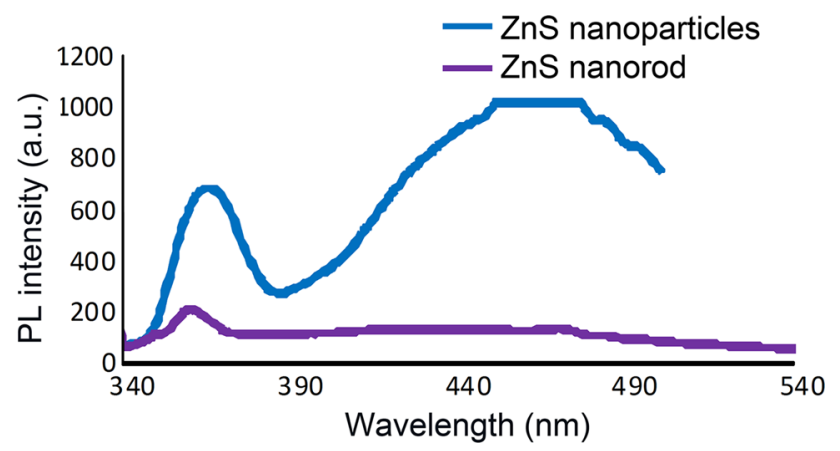

Fig. 5 PL spectra of ZnS nanopowder and nanorod

EIS using electrochemical workstation. The $\mathrm{ZnS}$ nanorod grown on the aluminum foil is used as working electrode; platinum wire and $\mathrm{Ag} / \mathrm{AgCl}$ are used as counter and reference electrodes, respectively [31]. The electrolyte solution used for obtaining EIS spectra is $3.5 \% \mathrm{NaCl}$ solution. Impedance values are measured in the frequency range from $1 \mathrm{MHz}$ to $1 \mathrm{~Hz}$ at room temperature.

It is evident from the Fig. 6a that the $\mathrm{ZnS}$ nanorod exhibits the charge transfer resistance value of about $651 \Omega$ and double-layer capacitance of $6.7 \mathrm{nF}$ with a characteristic semicircular-like impedance curve [30].
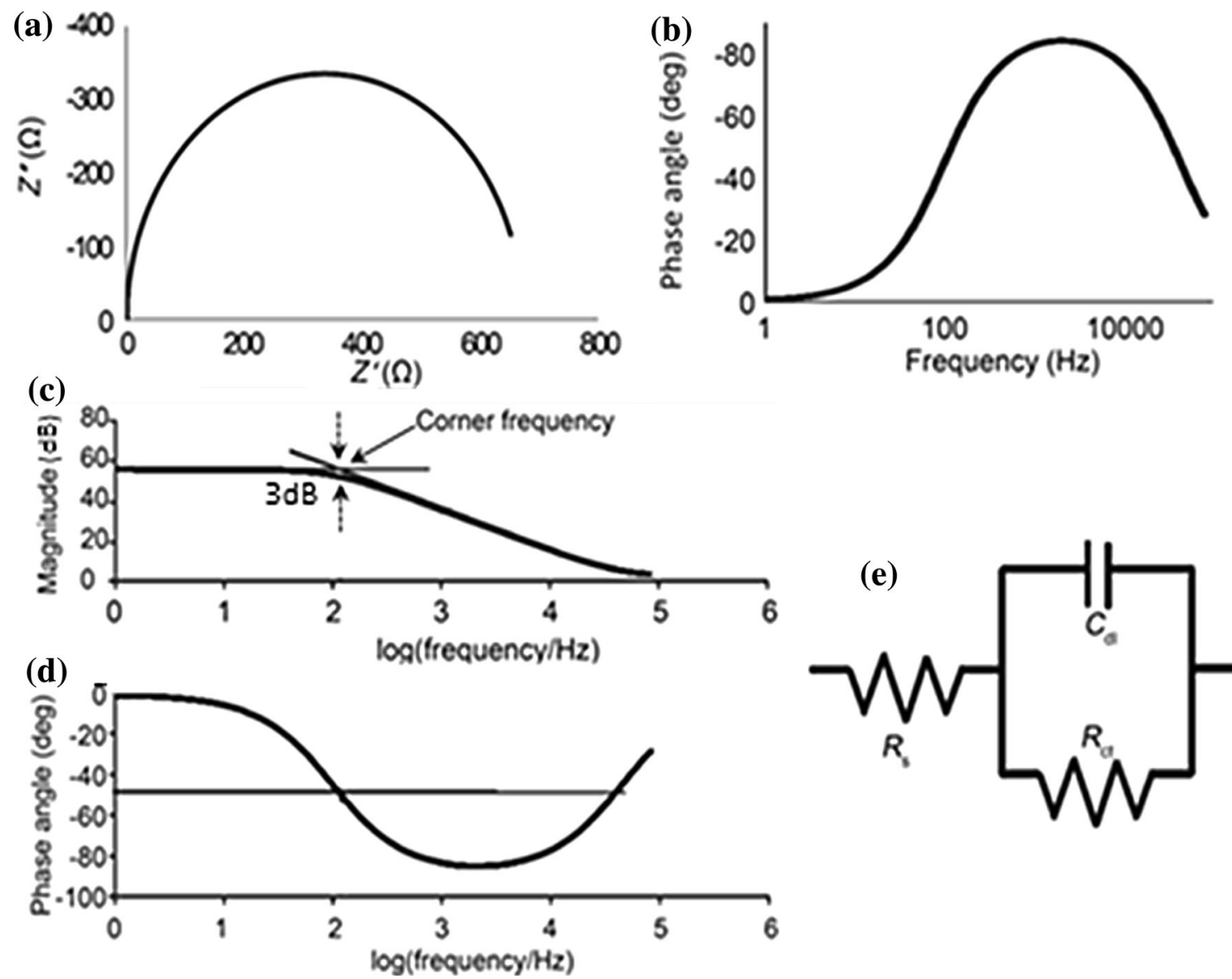
nanorod developed on aluminum foil, in which the phase angle versus frequency is plotted. The electron life time transport from conduction band to valence band for a semiconducting material is thickness dependent [17]. From AFM analysis, the thickness of the as-grown $\mathrm{ZnS}$ nanorod is found to be about $500 \mathrm{~nm}$. The semicircle form of $\mathrm{Ny}$ quist plot is a characteristic of the electron injection phenomenon taking place at the counter electrode-electrolyte interface. The recombination of electron residing on the conduction band to its valence band has been calculated using the Eq. (2). The electron life time transport value found for the $\mathrm{ZnS}$ nanorod is about $7.402 \times 10^{-5} \mathrm{~s}^{2}$. This value is less in comparison with the semi-conducting materials of quantum dot type [32].

Employing similar methodology which was reported in Ref. [33], the stability of $\mathrm{ZnS}$ nanorod also evaluated from the details of EIS data. The magnitude and phase angle plots of the open-loop frequency transfer function are evaluated from Bode diagrams illustrated in Fig. 6c and d. In particular, Fig. $6 \mathrm{c}$ is drawn by taking the magnitude in $y$ axis and the logarithmic frequency in $x$-axis. The highfrequency and low-frequency asymptotes are drawn from

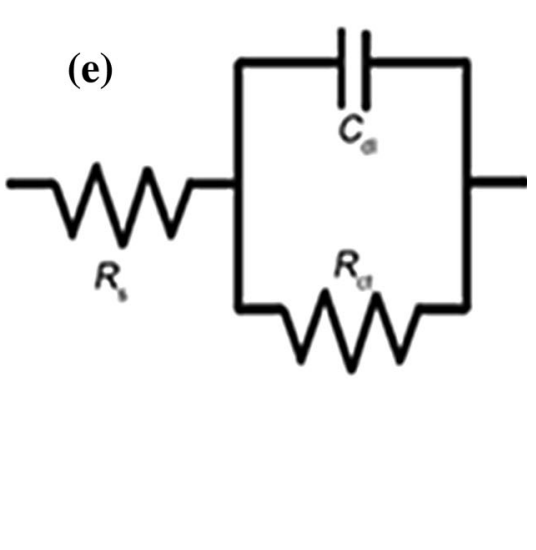

Fig. 6 Nyquist plot a, bode plot $\mathbf{b}$, magnitude plot $\mathbf{c}$, phase angle plot d, equivalent circuit model e of the $\mathrm{ZnS}$ nanorod 
both sides of the Bode plot-the point at which the two asymptotes intersect with each other is called as corner frequency or break frequency or break point. From the Fig. $6 c$, it is clear that the magnitude at the corner frequency is $60 \mathrm{~dB}$ (on $y$-axis). Based on the asymptotic plot approximation, the deviation from the corner frequency has been evaluated and found to be about $3 \mathrm{~dB}$. These results are in accordance with the fact that if a plot differs by a factor of $20 \times \log (1 / \sqrt{2})$ or $3 \mathrm{~dB}[34]$, then it can serve as an efficient electrode material for storing data.

Further, the Bode plot is reconstructed using phase angle and $\log$ frequencies, as represented in Fig. 6d. The corresponding phase angle at corner frequency is $-45^{\circ}$ which frequency is known as $\Phi_{\mathrm{gc}}$. From the equation $\gamma=180^{\circ}+\Phi_{\mathrm{gc}}$, the phase margin value is determined as $135^{\circ}$. The gain margin is calculated by the transfer function $(f(\omega))$ of the phase plot from the equivalent circuit model:

$f(\omega)=\frac{1017.95\left(1+1.5649 \times 10^{-6} j \omega\right)}{1+1.593 \times 10^{-3} j \omega}$,

where $j$ and $\omega$ are $\sqrt{-1}$ and frequency, respectively. The capacitance and the resistance values of the equivalent circuit model have been taken from the EIS data.

Obtained transfer function is converted into corresponding polar coordinates, and thus it is found to be equal to $-180^{\circ}$ as evaluated using the following equation:

$\tan ^{-1}\left(1.5649 \times 10^{-6}\right) \omega-\tan ^{-1}\left(1.593 \times 10^{-3}\right) \omega$
$\quad=-180^{\circ}$

Using the above expression, the value of $\omega$ is evaluated, and on substituting the value into the transfer function Eq. (3), it gives the gain margin value, which is found to be equal to infinity. From these results, it is apparent that the system, possessing both phase margin and gain margin as positive values, becomes electrically and electronically stable.

Figure 6e represents the equivalent circuit model drawn by considering solution resistance as $R_{\mathrm{s}}$, a double-layer capacitance as $C_{\mathrm{dl}}$, and a charge transfer or polarization resistance as $R_{\mathrm{ct}}$. The resistance was ascribed to the impedance of the charge transfer behavior of the $\mathrm{ZnS}$ coated aluminum foil due to the $3.5 \% \mathrm{NaCl}$-electrolyte interface. Higher capacitance $\left(C_{\mathrm{dl}}=6.7 \mathrm{nF}\right)$ value found in this system is attributable to the electron-storing capability of the $\mathrm{ZnS}$ nanorod. These results substantiate that the $\mathrm{ZnS}$ semiconducting material developed on aluminum foil have capacitance characteristics.

\section{Conclusions}

A novel method for the synthesis of $\mathrm{ZnS}$ nanorod using biotemplate method was established. XRD confirms the formation of $\mathrm{ZnS}$ of the corresponding materials. The morphology of $\mathrm{ZnS}$ nanorod was checked through SEM. The PL study of $\mathrm{ZnS}$ nanoparticle and nanorod indicated that the band-to-band transition and exciton recombination were size dependent. The EIS study shows that the capacitance and life time of electron were greater in $\mathrm{ZnS}$ nanorod coated over the aluminum substrate. AC impedance analysis proves that the developed nanorods possess electrical and electronic stabilities.

\section{References}

[1] X.S. Fang, T.Y. Zhai, U.K. Gautam, L. Li, L.M. Wu, Y. Bando, D. Golberg, Prog. Mater Sci. 56, 175 (2011)

[2] G.Z. Shen, D. Chen, Optoelectron. China 3, 125 (2010)

[3] D.H. Lee, J.Y. Jung, E.J. Bae, T.J. Lee, S.O. Ryu, J. Korean Phys. Soc. 53, 102 (2008)

[4] B.P. Singh, S.P. Singh, S. Vishnoi, R. Kumar, J. Optoelectron Biomed. Mater. 4, 29 (2012)

[5] R. John, S.S. Florence, Chalcogenide Lett. 7, 269 (2010)

[6] R.I. Sharma, Int. Multidiscip. Res. J. 1, 8 (2011)

[7] X.S. Fang, Y. Bando, M.Y. Liao, U.K. Gautam, C.Y. Zhi, B. Dierre, B.D. Liu, T.Y. Zhai, T. Sekiguchi, Y. Koide, D. Golberg, Adv. Mater. 21, 2034 (2009)

[8] X.S. Fang, Y. Bando, M.Y. Liao, T.Y. Zhai, U.K. Gautam, L. Li, Y. Koide, D. Golberg, Adv. Funct. Mater. 20, 500 (2010)

[9] L.F. Hu, J. Yan, M.Y. Liao, H.J. Xiang, X.G. Gong, L.D. Zhang, X.S. Fang, Adv. Mater. 24, 2305 (2012)

[10] N.T. Tuan, N.D. Trung-Kien, P.T. Huy, N.H. Tung, J. Surf. Sci. Nanotechnol. 9, 521 (2011)

[11] X.S. Fang, C.H. Ye, L.D. Zhang, Y.H. Wang, Y.C. Wu, Adv. Funct. Mater. 15, 63 (2005)

[12] X.S. Fang, Y.S. Bando, C.H. Ye, G.Z. Shen, D. Golberg, J. Phys. Chem. C 111, 8469 (2007)

[13] C. Jin, Y. Cheng, X. Zhang, W. Zhong, Y. Deng, C. Au, X. Wu, Y. Du, Cryst. Eng. Commun. 11, 2260 (2009)

[14] K. Kertesz, G. Molnar, Z. Vertesy, A.A. Koos, Z.E. Horvath, G.I. Mark, L. Tapaszto, Z. Balint, I. Tamaska, O. Deparis, J.P. Vigneron, L.P. Biro, Mater. Sci. Eng. B 149, 259 (2008)

[15] J.Y. Huang, X.D. Wang, Z.L. Wang, Nano Lett. 6, 2325 (2006)

[16] T. Kuwabara, M. Nakamoto, Y. Kawahara, T. Yamaguchi, K. Takahashi, J. Appl. Phys. 105, 1 (2009)

[17] J.J. Tian, R. Gao, Q.F. Zhang, S.G. Zhang, Y.W. Li, J.L. Lan, X.H. Qu, G.Z. Cao, J. Phys. Chem. 116, 18655 (2012)

[18] T.Y. Yesu, R. Anitha, B. Kavitha, Int. J. Appl. Sci. Eng. Res. 1, $282(2012)$

[19] S.C. Kim, J.W. Kim, H.S. Chung, D.H. Kim, K.H. Oh, J. Korean Phys. Soc. 55, 978 (2009)

[20] P. Sana, L. Hasmi, M.M. Malik, Int. Scholar. Res. Netw. 1, (2012)

[21] Y. Ding, S. Xu, Z.L. Wang, J. Appl. Phys. 106, 1 (2009)

[22] S. Velumani, J.A. Ascencio, Appl. Phys. A 79, 153 (2004)

[23] C.S. Tiwarya, P. Kumbhakara, A.K. Mitraa, K. Chattopadhyay, J. Lumin. 129, 1366 (2009)

[24] C.H. Ye, X.S. Fang, M. Wang, L.D. Zhang, J. Appl. Phys. 99, 063504 (2006)

[25] B. Bhattacharjee, C.H. Lu, Thin Solid Films 514, 132 (2006)

[26] Y. Zhao, J.M. Hong, J.J. Zhu, 270, 438 (2004)

[27] H.S. Moon, C.H. Nam, C.W. Kim, B.S. Kim, Mater. Res. Bull. 41, 2913 (2006) 
[28] N. Soltani, A. Dehzangi, E. Saion, B.Y. Majlis, M.R. Zare, A. Kharazmi, M. Navasery, Chalcogenide Lett. 10, 27 (2013)

[29] M. Ahmad, K. Rasool, M.A. Rafiq, M.M. Hasan, Paper presented at Communications and Photonics Conference (SIECPC), Saudi International, 24-26 April, (2011)

[30] J.R. Macdonold, Annu. Biomed. Eng. 20, 289 (1992)

[31] R. Hrdy, H. Kynclova, J. Drbohlavova, V. Svatos, J. Chomoucka, J. Prasek, P. Businova, J. Pekarek, L. Trnkova, R. Kizek, J. Hubalek, Int. J. Electrochem. Sci. 8, 4384 (2013)
[32] A. Nakhmani, M. Lichtsinder, E. Zeheb, paper presented at IEEE 24th Convention of Electrical and Electronics Engineers in Israel, 26 November (2006)

[33] R. Mancini, Feedback and Stability Theory, Excerpted from Op Amps for Everyone, (2006) http://www.ti.com/lit/ml/sloa077/ sloa077.pdf. Accessed 12.12.2013

[34] Zoher Z. Karu, Signals and Systems Made Ridiculously Simple, ZiZi Press, Huntville, AL35802, USA, 1995, Chapter 6, Page No. 26 